\title{
GCU
}

Glasgow Caledonian

University

University for the Common Good

\section{Estimating the number of injecting drug users in scotland's HCV-diagnosed population using capture-recapture methods}

McDonald, Scott A.; Schnier, Christian; Hutchinson, Sharon; McLeod, Allan; Goldberg, David

Published in:

Epidemiology and Infection

DOI:

$10.1017 / \mathrm{S} 0950268813000617$

Publication date:

2014

Document Version

Author accepted manuscript

Link to publication in ResearchOnline

Citation for published version (Harvard):

McDonald, SA, Schnier, C, Hutchinson, S, McLeod, A \& Goldberg, D 2014, 'Estimating the number of injecting drug users in scotland's HCV-diagnosed population using capture-recapture methods', Epidemiology and Infection, vol. 142, no. 1, pp. 200-207. https://doi.org/10.1017/S0950268813000617

\section{General rights}

Copyright and moral rights for the publications made accessible in the public portal are retained by the authors and/or other copyright owners and it is a condition of accessing publications that users recognise and abide by the legal requirements associated with these rights.

Take down policy

If you believe that this document breaches copyright please view our takedown policy at https://edshare.gcu.ac.uk/id/eprint/5179 for details

of how to contact us. 
Estimating the number of injecting drug users in Scotland's HCV-diagnosed population using capture-recapture methods

S. A. McDonald ${ }^{1,2}$, S. J. Hutchinson ${ }^{1,2}$, C. Schnier ${ }^{1,2}$, A. McLeod ${ }^{1}$, D. J. Goldberg ${ }^{1}$

${ }^{1}$ Health Protection Scotland, Meridian Court, 5 Cadogan Street, Glasgow G2 6QE, Scotland, UK ${ }^{2}$ Department of Mathematics and Statistics, University of Strathclyde, 26 Richmond Street, Glasgow G1 1XH, Scotland, UK

\section{Corresponding author}

S. A. McDonald

Health Protection Scotland

Meridian Court, 5 Cadogan Street,

Glasgow G2 6QE, Scotland, UK

Email: smcdonald4@nhs.net

Tel: +44 1413001106

Fax: +44 1413001170

Short running head Capture-recapture methods for IDU risk

Word count: 2,779 words excluding references and tables 


\section{SUMMARY}

In countries maintaining national hepatitis $\mathrm{C}$ virus $(\mathrm{HCV})$ surveillance systems, a substantial proportion of individuals report no risk factors for infection. Our goal was to estimate the proportion of diagnosed HCV antibody-positive persons in Scotland (1991-2010) who likely acquired infection through injecting drug use (IDU), by combining data on IDU risk from four linked data sources using log-linear capture-recapture methods. Of $25521 \mathrm{HCV}$-diagnosed individuals, 14836 (58\%) reported IDU risk with their HCV diagnosis. Log-linear modeling estimated a further 2,484 HCV-diagnosed individuals with IDU risk, giving an estimated prevalence of 83 (95\% CI: 81-87\%). Stratified analyses indicated variation across birth cohort, with estimated prevalence as low as $49 \%$ in persons born before 1960 and greater than $90 \%$ in those born since 1960. These findings provide public-health professionals with a more complete profile of Scotland's HCV-infected population in terms of transmission route, which is essential for targeting educational, prevention and treatment interventions. 


\section{INTRODUCTION}

Injecting drug use (IDU) represents the most common risk factor for $\mathrm{HCV}$ infection throughout the industrialized world.[1] However, a substantial proportion of persons who test HCV antibody-positive report no risk factors for acquiring infection. In the United States, $30 \%$ of persons with acute HCV during 1991-1995 denied a specific exposure associated with becoming infected during the six months preceding illness onset, although over half of these reported a history of drug use.[2] In England and Wales, 71\% (35598/49819) of confirmed HCV infections during 1992-2004 lacked risk factor information.[3] Sentinel surveillance of acute HCV infection in the United States indicates sexual risk behaviour as a probable route of infection in a significant minority of cases [4,5]. Either the potential risk factors for HCV acquisition were not carefully elicited in these studies, or there was a significant undefined source of viral transmission. A study in the United States showed that the route of HCV acquisition could be delineated in $88 \%$ of HCV chronically infected patients using a systematic interview approach; in nearly all cases, the initially unreported risk factor for HCV transmission was a remote history of injecting drug use.[6]

Deriving an estimate of the percentage of HCV-diagnosed persons with IDU risk from the observed data can be problematic (i.e., subject to bias) if there is a large amount of missing risk information, which is the case for the national HCV diagnosis database held by Health Protection Scotland (HPS), a population-wide record of all individuals testing HCV antibodypositive since testing commenced in 1991 . As at the end of 2009, 35\% of records lacked data on risk factor(s), and of those with risk information, current/former IDU was specified for 89\%.[7] 
An unknown percentage of persons with missing risk data will have acquired their infection through IDU.

In the current study, we combined data on injecting drug use history available from four other data sources - HIV testing, hospital discharges, deaths, treatment for drugs misuse - with that on the HCV diagnoses database, using record-linkage methods to identify individuals observed across sources. The total number of HCV-diagnosed persons with IDU risk was then estimated using capture-recapture statistical methods,[8] originally developed for counting animal populations. Thus, the purpose of our study was to estimate of the proportion of HCV antibody-positive and diagnosed persons who were likely to have acquired their infection through injecting drug use, which will provide public health policy-makers with a more accurate demographic picture of Scotland's HCV-infected population, and will consequently inform on resource allocation for prevention, treatment and care.

\section{METHODS}

The IDU status of HCV-diagnosed persons was sourced from five databases (i.e. HCV diagnoses, HIV tests, treatment for drug misuse, hospital discharges, and deaths databases). Data on opiate use rather than injecting drug use per se was available from the latter two databases, and served as a proxy indicator for IDU.

The HCV Diagnosis database, maintained by HPS, records all persons who have been diagnosed HCV positive (defined as laboratory detection of HCV antibody or a positive PCR test result) in Scotland since testing began in 1991. The database contains the following non- 
named information: surname Soundex code (multiple surnames possible; for instance, following marriage), forename initial, date of birth, sex, postcode district of residence, hospital/clinic number (generated from GUM clinic/hospital referrals only), and data concerning risk activities; as at 31 December 2009, this database contained records for 27183 persons.

The second data source used was the national HIV test database, also held by HPS. It records all HIV tests conducted within Scotland, excluding routine screening (e.g., antenatal, renal, travel/insurance), and persons under 15 years of age. Data is provided by all NHS laboratories in Scotland that perform HIV testing. Individual records on this database contain the following non-named information: sex, date of birth, surname and forename initials, health board of residence (HB; NHS Scotland administrative area) and a hospital/clinic number generated from GUM clinic/hospital referrals only, as well as data concerning risk activities. HIV Test records were mapped to distinct individuals using a deterministic approach (i.e., the procedure required a complete match on either the set of identifiers sex, date of birth, and initials, or the set of sex, date of birth, and hospital/clinic number). The HIV Test database contained records for 523251 HIV tests conducted between 1 January 1988 and 31 December 2009 (including the testing of some stored sera back to 1980). Internal linkage resulted in records for 412994 distinct HIVtested individuals. Linkage between the HCV Diagnosis and HIV Test databases was also performed in-house at HPS using deterministic methods (i.e., a complete match was required on the identifier set (i) sex, date of birth, and initials; or, if initials were missing, the set (ii) sex, date of birth, and hospital/clinic number).

The Scottish Morbidity Records (SMR01) is an episode-based patient record of all acute inpatient and day case hospital discharges from non-obstetric, non-psychiatric specialities. Information Services Division (ISD) routinely combines SMR01 data with death registrations 
held by the General Register Office for Scotland to form a linked dataset; the identifiers sex, date of birth, initials, and surname Soundex were available. We treated this linked dataset (of hospital and death records) as a single data source for the capture-recapture analysis. Linkage of records between the HCV Diagnosis database and the hospitalisation/deaths dataset was carried out by ISD using probabilistic record-linkage techniques, [9] which allow for matches using incomplete identifiers. All hospital and death records (for the period 1 January 1981 to 31 December 2009) that had linked to HCV diagnosed persons were provided for analysis. The final data source used was the Scottish Drug Misuse Database (SDMD), also held by ISD. The SDMD is a record of current/former drug users in contact with drug treatment and support services, including general practitioners, hospitals, specialist drug clinics, and non-statutory agencies. These agencies report information on new contacts (defined as first presentation or repeat presentation if it has been at least six months since last attendance) to the SDMD. The SDMD contains limited identifying information: sex, date of birth, forename initial, first and fourth letter of surname, and postcode sector of residence. Data were available from 1 April 1996 through 31 December 2009, containing 76364 records representing 28601 distinct individuals. Data-linkage between the SDMD and HCV Diagnosis databases was performed by ISD using probabilistic methods.

After exclusion of 1568 persons with insufficient identifiers (defined as missing date of birth and two of: sex, initials, surname Soundex), 25615 records on the HCV Diagnosis database were available for linkage to the other databases. Approval for this linkage exercise was provided by the NHS National Services Scotland Privacy Advisory Committee. The study population was further restricted to all records with non-missing sex data, leaving 25521 for analysis. 


\section{Definition of IDU risk}

An individual on the HCV Diagnosis or HIV Test databases was considered to have IDU risk if IDU was listed as a risk factor for acquiring infection.

In the SMR01/death registrations linked dataset, both hospital discharge diagnosis and cause of death codes use the International Classification of Diseases (ICD) Ninth Revision for events occurring before 2000, and the Tenth Revision for the period 2000-2009. IDU risk was inferred if the discharge/death record contained a code for opiate use, i.e. any of the ICD-9 codes 304.0 'Opioid type dependence' or 304.7 'Combinations of opioid type drug with any other drug dependence', or the ICD-10 codes F11.0-F11.9 'Mental \& behavioural disorders due to use of opioids'.

IDU risk for an individual on the SDMD was defined according to self-report: if at any attendance at drug services the client reported having either 'injected in the previous month' or 'injected in past/not previous month', he/she was classified as having IDU risk.

\section{Statistical methods}

Log-linear modelling[8] was used to analyse the overlap in the number of HCV-diagnosed persons with IDU risk among the four data-sources (HCV diagnosis, HIV test, hospital/death and SDMD) and estimate the total population size (i.e., the number of HCV diagnosed IDUs including those who are unknown). Backwards stepwise regression was used to find a model which adequately described the data with the least number of parameters; 2-way and 3-way interaction terms were removed from the model specification if the Akaike Information Criterion (AIC) difference was $<2$ compared with the model including the interaction term. 
Confidence intervals were determined using the profile likelihood. Model fitting was done using the Rcapture package [10] for R statistical software.[11]

Stratified log-linear analyses were conducted according to four covariates: sex, birth cohort $(<1960,1960-1969,1970-1979,1980+)$, health board of residence (Greater Glasgow \& Clyde, and all other), and calendar year period of HCV diagnosis (<1995, 1995-99, 2000-04, 2005-09).

Additional analyses were conducted restricting to those HCV-diagnosed who had non-IDU risk and to those for whom risk activity leading to infection was unknown. In order to investigate the possibility that there might not have been sufficient time for those persons diagnosed with $\mathrm{HCV}$ near the end of the study period to be 'captured' on the other data sources, in a sensitivity analysis we restricted the inclusion period to HCV diagnoses made up to 31 December 2006 (with the other data sources censored as before, at end 2009).

\section{RESULTS}

\section{Study population}

Among all HCV-diagnosed persons, 58\% (14836/25521) reported IDU risk at the time of their HCV diagnosis, 7.3\% (1862/25521) reported a non-IDU risk (e.g. blood factor/transfusion) and for $35 \%$ the risk factor(s) for acquiring infection were unknown. Among those with a reported risk(s) on the HCV Diagnosis database, 89\% (14836/16698) were current/former IDUs. The majority of HCV-diagnosed persons were male (68\%), and were born during the 1960s and 1970s (70\%) (Table 1).

\section{IDU risk from other data sources}


Of the $25521 \mathrm{HCV}$-diagnosed persons, 38\%, 49\% and 30\% were identified as having an IDU risk on the HIV test, SDMD and hospital/deaths databases, respectively (Table 1). Among HCVdiagnosed persons who had reported a non-IDU risk at the time of HCV diagnosis, $11 \%$ (200/1862), 16\% (294/1862) and 9\% (171/1862) were identified as having IDU risk on the HIV test, SDMD and hospitalisation/deaths databases, respectively. The distribution of covariates across data sources varied somewhat for birth cohort (Table 1). Twenty per cent of all HCVdiagnosed persons were born prior to the 1960s, compared to only $6 \%$ of those identified with IDU risk on the SDMD, and 10\% of those with IDU risk on either the HCV Diagnosis or hospitalisation/deaths data sources. Across data sources, the proportion of individuals with an IDU risk residing in Greater Glasgow \& Clyde health board was relatively constant, at $40 \%$ to $46 \%$, as was the proportion of males, at $69 \%$ to $74 \%$.

Of the $25521 \mathrm{HCV}$-diagnosed persons, 18782 (74\%) had IDU risk recorded on at least one of the four data-sources (i.e. on either the HCV diagnosis, HIV test, SDMD or hospital/deaths databases). Overall sensitivity of the HCV Diagnosis database for recording IDU risk (with respect to the 'gold standard' of IDU status determined from any of the four linked datasources) was 79.0\%, which varied according to covariate level (Table 1).

\section{Log-linear modelling}

The log-linear model fitting procedure retained all 2-way and 3-way interaction terms, and predicted a further 2484 IDUs not identified from the four data sources (Table 2) for an estimated total of 21266 IDUs (95\% CI: 20582 3-22140); this corresponded to an estimated IDU prevalence among all HCV-diagnosed persons of 83.3\% (21266/25521). The sensitivity analysis, 
in which individuals diagnosed after 31 December 2006 were excluded (resulting $n=20612$ ), indicated a slightly higher estimated IDU prevalence (84.8\%).

As a validity check, we also estimated IDU population size for only those persons with IDU risk present on the HCV Diagnosis database $(n=14836)$ from the other three data sources; the estimated total number of IDUs was 14336 (95\% CI: 14069-14629) and estimated IDU prevalence for this group of known IDUs was $96.6 \%$, slightly less than the $100 \%$ one would expect. However, this analysis was necessarily based on the three data sources with the most impoverished IDU risk information. An additional analysis, excluding 1872 persons whose HCV diagnosis record specified a non-IDU risk activity (reducing to $N=23659$ ), also resulted in fewer estimated HCV-diagnosed IDUs: 19935 (95\% CI: 19724-20170) compared with 21266 (the latter value estimated in Table 2).

Stratified log-linear models fitted to the data according to sex, birth cohort, period of HCV diagnosis, and HB group indicated substantial variation in estimated IDU prevalence (Table 3). Prevalence was lowest for the oldest cohort (born before 1960, 49.4\%) and highest for individuals born 1970-79 (93.4\%). Estimated prevalence was also highest for males (84.6\%) and for persons diagnosed with HCV during 1995-1999 (91.5\%).

\section{DISCUSSION}

This application in Scotland is the first to demonstrate the use of log-linear modelling, based on capture-recapture data from four linked sources, to estimate the proportion of IDUs among HCV diagnosed persons. The estimated prevalence of current/former IDU was 83\% in Scotland's 
HCV-diagnosed population, substantially higher than the $58 \%$ who had reported IDU as risk activity. This estimated prevalence was somewhat lower than an estimate of IDU prevalence derived from the $65 \%$ of the study population with reported risk factor(s) (89\%). However, if individuals diagnosed with HCV in the three most recent years of the study period are excluded (to allow more opportunity for 'capture' by the other data sources), the prevalence was estimated at $85 \%$. The latter figure is closely comparable to the value $(87 \%)$ obtained from laboratory surveillance in England \& Wales in 1992-2004.[3]

Stratified analyses indicated that estimated IDU prevalence was lowest (67\%) among individuals diagnosed with HCV before 1995; this is consistent with an over-representative contribution to the early growth of the database from persons with blood clotting disorders. IDU prevalence was highest among those born in the 1960s and 1970s, reflecting the age-groups in Scotland in which problem drug use is the most prevalent,[12] and in which risky injecting practices are frequent.[13] Estimated IDU prevalence was also higher for male, compared with female, HCV diagnosed persons ( $86 \%$ and $78 \%$, respectively), which may be due to male sex being an independent risk factor for acquiring $\mathrm{HCV}$ infection among IDUs, leading to more male than female HCV-infected IDUs appearing on the HCV Diagnosis database.

The estimated sensitivity of the risk information field in the HCV Diagnosis database also varied according to birth cohort, and period of diagnosis, with the lowest accuracy observed for the youngest cohort (74\%) and most recent $\mathrm{HCV}$ diagnosis period (69\%); the latter finding is consistent with there sometimes being a short lag in the collection and recording of risk activity data on the HCV Diagnosis database.. 
The only other study we are aware of in which the number of HCV-infected IDUs was estimated using capture-recapture methods was conducted in Porto Alegre, Brazil.[14] In this study, the total number of IDUs attending syringe-exchange programmes was estimated based on two interviewed samples approximately one month apart, and then overall HCV seroprevalence in this population (53\%) was assumed for the estimated total IDUs (168/317). However, the proportion of IDU risk among HCV-diagnosed persons was not estimated in the study.

Although the application of capture-recapture and log-linear modelling methods to epidemiological questions has certain strengths, it has a number of limitations.

Firstly, we have had to assume that all four data sources reflect the same (closed) population. In reality, especially over the long study period, new individuals enter and others leave the population, through initiation of drug use, and death. Secondly, within a given data source, each IDU was assumed to have the same chance of being included (i.e., to have the same 'catchability'). Although we have attempted to address the issue of heterogeneity in being observed within a given data source by conducting stratified analyses, an unknown degree of variability will remain. Subgroups with low catchability might bias estimates of the prevalence of IDU within the HCV-diagnosed population downwards. Finally, violation of the assumed high accuracy of the record-linkage methods could also result in bias. Although the probabilistic methods used by ISD to link HCV Diagnosis with the SMR01/deaths linked dataset have historically low false positive and false negative rates $(<5 \%[9])$, accuracy estimates were not available for the other linkages performed.

In conclusion, the proportion of Scotland's HCV-diagnosed population who were estimated to have acquired their infection through injecting drug use was smaller than if estimated from only 
the data with non-missing risk information, but once opportunity for capture on the other data sources was increased, the proportion with IDU risk was more similar. Information on the route by which HCV infection is acquired is essential when targeting risk groups with educational and prevention interventions, and is also useful for governmental and public health professionals who develop policy and allocate funding for treatment and care. Our results - indicating a similar high prevalence of IDU among HCV-diagnosed individuals with missing data on risk activities, as among those with risk activity reported - provide evidence that efforts to prevent and treat HCV infection should focus on this risk group.

\section{ACKNOWLEDGEMENTS}

We thank Glenn Codere and Amanda Weir for their assistance with the HIV Test database,

Information Services Division, NSS Scotland, for conducting the probabilistic record-linkage and the provision of hospitalisation/deaths and SDMD datasets, and the various laboratories across Scotland for providing national data on HCV diagnoses and HIV testing.

\section{DECLARATION OF INTERESTS}

None. 


\section{REFERENCES}

[1] Di Bisceglie AM. Hepatitis C. Lancet 1998; 351: 351-355.

[2] Alter MJ. Epidemiology of hepatitis C. Hepatology 1997; 26: 62S-65S.

[3] Gungabissoon U, Balogun MA, Ramsay ME. Hepatitis C virus: laboratory surveillance in England and Wales, 1992-2004. Epidemiology and Infection 2007; 135: 541-548.

[4] Wasley A, Grytdal S, Gallagher K. Surveillance for acute viral hepatitis -- United States, 2006. MMWR 2008; 57: 1-24.

[5] Williams IT, et al. Incidence and transmission patterns of acute hepatitis C in the United States, 1982-2006. Archives of Internal Medicine 2011; 171: 242-248.

[6] Flamm S, Parker R, Chopra S. Risk factors associated with chronic hepatitis C virus infection: limited frequency of an unidentified source of transmission. American Journal of Gastroenterology 1998; 93: 597-600.

[7] Health Protection Scotland (HPS). Surveillance of known hepatitis C antibody positive cases in Scotland: Results to 31 December 2009. HPS Weekly Report: Health Protection Scotland 2010; 44: $175-178$.

[8] Cormack RM. Log-linear models for capture-recapture. Biometrics 1989; 45: 395-413.

[9] Kendrick S, Clarke J. The Scottish Record Linkage System. Health Bulletin (Edinburgh) 1993; 51: 72-79.

[10] Baillargeon S, Rivest L. Rcapture: Loglinear Models for Capture-Recapture in R. Journal of Statistical Software 2007; 19.

[11] R Development Core Team. R: A language and environment for statistical computing. Vienna, Austria: R Foundation for Statistical Computing, 2008.

[12] Hay G, et al. Estimating the national and local prevalence of problem drug misuse in 
Scotland: Executive Report. 2009.

[13] University of the West of Scotland, Health Protection Scotland and West of Scotland Specialist Virology Centre. The Needle Exchange Surveillance Initiative (NESI): Prevalence of $\mathrm{HCV}, \mathrm{HIV}$ and injecting risk behaviours among injecting drug users attending needle exchanges in Scotland, 2008/2009. 2010.

[14] Caiaffa WT, et al. Estimation of the number of injecting drug users attending an outreach syringe-exchange program and infection with human immunodeficiency virus (HIV) and hepatitis C virus: the AjUDE-Brasil project. Journal of Urban Health 2003; 80: 106-114. 
Table 1. Study population (first column; $n=25,521$ ) and distribution of IDUs identified via record-linkage in each of the four data sources according to sex, birth cohort, health board of residence and calendar year period of HCV diagnosis. The sensitivity of IDU status on the HCV Diagnosis database is computed by considering the IDUs identified by aggregating all four data sources as the 'gold standard'.

All HCV HCV-diagnosed cases with IDU risk on the following data sources:

\begin{tabular}{|c|c|c|c|c|c|c|c|c|c|c|c|c|}
\hline \multirow{2}{*}{\multicolumn{2}{|c|}{ Covariate }} & \multicolumn{2}{|c|}{ Diagnosed } & \multicolumn{2}{|c|}{ (a) HCV Diag } & \multicolumn{2}{|c|}{ (b) HIV Tes } & \multicolumn{2}{|c|}{ t (c) SDMD } & \multicolumn{2}{|c|}{ (d) SMR01/GROS } & \multirow{2}{*}{$\begin{array}{c}\text { Sensitivity } \\
\%\end{array}$} \\
\hline & & $N$ & $\%$ & $\mathrm{~N}$ & $\%$ & $\mathrm{~N}$ & $\%$ & $\mathrm{~N}$ & $\%$ & $\mathrm{~N}$ & $\%$ & \\
\hline All & & 25521 & - & 14836 & - & 9761 & - & 12410 & - & 7625 & - & 79.0 \\
\hline \multirow[t]{2}{*}{ Sex } & Female & 8177 & 32.0 & 4415 & 29.8 & 2524 & 25.9 & 3806 & 30.7 & 2361 & 31.0 & 77.5 \\
\hline & Male & 17344 & 68.0 & 10421 & 70.2 & 7237 & 74.1 & 8604 & 69.3 & 5264 & 69.0 & 79.6 \\
\hline \multirow{4}{*}{$\begin{array}{l}\text { Birth } \\
\text { cohort }\end{array}$} & $<1960$ & 5168 & 20.2 & 1530 & 10.3 & 889 & 9.1 & 790 & 6.4 & 771 & 10.1 & 78.1 \\
\hline & $1960-69$ & 8894 & 34.8 & 5557 & 37.5 & 3637 & 37.3 & 4260 & 34.3 & 2843 & 37.3 & 81.2 \\
\hline & $1970-79$ & 8812 & 34.5 & 6119 & 41.2 & 4148 & 42.5 & 5706 & 46.0 & 3170 & 41.6 & 78.6 \\
\hline & $1980+$ & 2647 & 10.4 & 1630 & 11.0 & 1087 & 11.1 & 1654 & 13.3 & 841 & 11.0 & 74.0 \\
\hline \multirow{2}{*}{$\begin{array}{l}\text { Health } \\
\text { board }\end{array}$} & GGC & 10679 & 41.8 & 6743 & 45.5 & 4090 & 41.9 & 5537 & 44.6 & 3045 & 39.9 & 82.5 \\
\hline & Other & 14842 & 58.2 & 8093 & 54.5 & 5671 & 58.1 & 6873 & 55.4 & 4580 & 60.1 & 76.3 \\
\hline \multirow{4}{*}{$\begin{array}{l}\text { Period } \\
\text { of HCV } \\
\text { diagn. }\end{array}$} & $<1995$ & 2290 & 9.0 & 1014 & 6.8 & 686 & 7.0 & 585 & 4.7 & 584 & 7.7 & 83.7 \\
\hline & 1995-99 & 7163 & 28.1 & 4597 & 31.0 & 2993 & 30.7 & 3388 & 27.3 & 2329 & 30.5 & 84.3 \\
\hline & 2000-04 & 8168 & 32.0 & 5341 & 36.0 & 3518 & 36.0 & 4436 & 35.7 & 2642 & 34.6 & 82.4 \\
\hline & 2005-09 & 7900 & 31.0 & 3884 & 26.2 & 2564 & 26.3 & 4001 & 32.2 & 2070 & 27.1 & 68.9 \\
\hline
\end{tabular}

Note. HCV Diag refers to the HCV Diagnosis database; SMR01/GROS refers to hospital/deaths data; GGC refers to Greater Glasgow \& Clyde health board. 
Table 2. IDU risk status of 25,521 individuals diagnosed with HCV in Scotland to 31 Dec 2009, according to four data-sources ((A) HCV Diagnosis, (B) HIV Test, (C) combined hospital discharges/deaths, and (D) SDMD). 95\% confidence intervals were derived using the profile likelihood.

\begin{tabular}{|c|c|c|c|c|}
\hline \multicolumn{4}{|c|}{$\begin{array}{l}\text { IDU risk identified on } 1 \text { or more of the following } 4 \text { data sources } \\
\qquad(\mathrm{Y}=\mathrm{yes} ; \mathrm{N}=\text { no) }\end{array}$} & \multirow[t]{3}{*}{$\begin{array}{l}\text { Number of HCV- } \\
\text { diagnosed IDUs }\end{array}$} \\
\hline (A) & (B) & (C) & (D) & \\
\hline HCV Diagnosis & HIV Test & SMR01/ Deaths & SDMD & \\
\hline $\mathrm{Y}$ & $\mathrm{Y}$ & $\mathrm{Y}$ & Y & 2814 \\
\hline $\mathrm{N}$ & $\mathrm{Y}$ & $\mathrm{Y}$ & Y & 513 \\
\hline Y & $\mathrm{N}$ & Y & Y & 1914 \\
\hline $\mathrm{N}$ & $\mathrm{N}$ & $\mathrm{Y}$ & Y & 801 \\
\hline Y & $\mathrm{Y}$ & $\mathrm{N}$ & Y & 2686 \\
\hline $\mathrm{N}$ & $\mathrm{Y}$ & $\mathrm{N}$ & Y & 520 \\
\hline Y & $\mathrm{N}$ & $\mathrm{N}$ & Y & 2083 \\
\hline $\mathrm{N}$ & $\mathrm{N}$ & $\mathrm{N}$ & $\mathrm{Y}$ & 1079 \\
\hline Y & $\mathrm{Y}$ & $\mathrm{Y}$ & $\mathrm{N}$ & 623 \\
\hline $\mathrm{N}$ & $\mathrm{Y}$ & Y & $\mathrm{N}$ & 126 \\
\hline Y & $\mathrm{N}$ & Y & $\mathrm{N}$ & 526 \\
\hline $\mathrm{N}$ & $\mathrm{N}$ & $\mathrm{Y}$ & $\mathrm{N}$ & 308 \\
\hline Y & $\mathrm{Y}$ & $\mathrm{N}$ & $\mathrm{N}$ & 1880 \\
\hline $\mathrm{N}$ & $\mathrm{Y}$ & $\mathrm{N}$ & $\mathrm{N}$ & 599 \\
\hline$Y$ & $\mathrm{~N}$ & $\mathrm{~N}$ & $\mathrm{~N}$ & 2310 \\
\hline $\mathrm{N}$ & $\mathrm{N}$ & $\mathrm{N}$ & $\mathrm{N}$ & - \\
\hline \multicolumn{4}{|c|}{$\begin{array}{l}\text { Total number of HCV-diagnosed persons identified with IDU risk from the four } \\
\text { data-sources (A) to (D) }\end{array}$} & 18,782 \\
\hline
\end{tabular}

Estimated number of HCV-diagnosed persons with IDU risk from log-linear 2,484 modeling, but were not identified as such from the four data-sources (A) to (D)

Estimated total number of HCV-diagnosed IDUs

Note. $\mathrm{Y}=$ yes; $\mathrm{N}=$ no. Backward step-wise fitting of log-linear model (residual deviance of 0 on 0 residual df) included main effects for data-sources (A) HCV diagnoses, (B) HIV test, (C) hospital discharge/deaths and (D) SDMD, and all 2-way and 3-way interactions between data-sources. 
Table 3. Results of log-linear modelling and estimated prevalence of IDU in HCV-diagnosed persons, for both full and stratified datasets; the latter were stratified by sex, birth cohort and period of HCV diagnosis. $95 \%$ confidence intervals were derived using the profile likelihood.

\begin{tabular}{|c|c|c|c|c|c|c|c|c|}
\hline & & & IDU risk, & rom & IDL & J risk, total & Estimated & Saturated \\
\hline & & $\mathrm{N}$ & data sour & es (\%) & $\mathrm{N}$ & $95 \%$ CI & prevalence & model? \\
\hline Full data & & 25521 & 18782 & (73.6) & 21266 & $20582-22140$ & $83.3 \%$ & Y \\
\hline Stratified & lysis & & & & & & & \\
\hline Sex & Female & 8177 & 5694 & (69.6) & 6230 & $6130-6341$ & $76.2 \%$ & $\mathrm{~N}^{\mathrm{a}}$ \\
\hline & Male & 17344 & 13088 & (75.5) & 14669 & $14235-15229$ & $84.6 \%$ & $\mathrm{~N}^{\mathrm{b}}$ \\
\hline Birth & $<1960$ & 5168 & 1958 & (37.9) & 2554 & $2366-2801$ & $49.4 \%$ & $\mathrm{~N}^{c}$ \\
\hline cohort & $1960-69$ & 8894 & 6841 & (76.9) & 8089 & $7575-8865$ & $91.0 \%$ & Y \\
\hline & $1970-79$ & 8812 & 7781 & (88.3) & 8226 & $8146-8316$ & $93.4 \%$ & $\mathrm{~N}^{\mathrm{d}}$ \\
\hline & $1980+$ & 2647 & 2202 & (83.2) & 2405 & $2347-2474$ & $90.8 \%$ & $\mathrm{Ne}^{\mathrm{e}}$ \\
\hline Health & GG & 10679 & 8174 & (76.5) & 8856 & $8698-9045$ & $82.9 \%$ & $\mathrm{~N}^{\mathrm{f}}$ \\
\hline Board & Other & 14842 & 10608 & (71.5) & 12573 & 11914-13491 & $84.7 \%$ & $\mathrm{Y}$ \\
\hline Period & $<1995$ & 2290 & 1211 & (52.9) & 1411 & $1304-1600$ & $61.6 \%$ & $\mathrm{Ng}$ \\
\hline of $\mathrm{HCV}$ & 1995-99 & 7163 & 5454 & (76.1) & 6554 & $6039-7389$ & $91.5 \%$ & $\mathrm{Y}$ \\
\hline diag. & 2000-04 & 8168 & 6479 & (79.3) & 6912 & 6830-7004 & $84.6 \%$ & $\mathrm{~N}^{\mathrm{h}}$ \\
\hline & 2005-09 & 7900 & 5638 & (71.4) & 6192 & $6075-6326$ & $78.4 \%$ & $\mathrm{~N}^{\mathrm{i}}$ \\
\hline
\end{tabular}

Note. ID'd=Identified; $Y=y e s ; \mathrm{N}=$ no; $\mathrm{CI}=$ confidence interval; GG=Greater Glasgow \& Clyde. 'Saturated model?' $=$ Y refers to a log-linear model in which all main effects and all possible 2- and 3-way interactions were retained after applying the backward stepwise selection. ${ }^{a}$ All 2-way interactions only (residual $\mathrm{df}=$

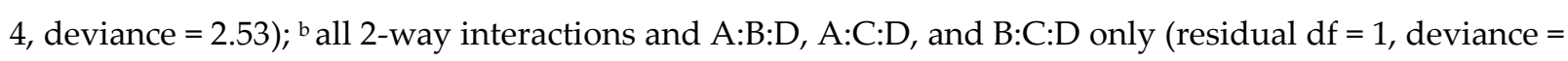
1.70); c all 2-way interactions and A:C:D only (residual $\mathrm{df}=3$, deviance $=1.66$ ); ${ }^{\mathrm{d}}$ all 2-way interactions only (residual $\mathrm{df}=4$, deviance $=1.90)$; ${ }^{\mathrm{e}}$ all 2-way interactions except $\mathrm{A}: \mathrm{C}$ and $\mathrm{B}: \mathrm{C}$ (residual $\mathrm{df}=6$, deviance $=$ 3.47); ${ }^{\mathrm{f}}$ all 2-way interactions and A:B:C and A:C:D only (residual $\mathrm{df}=2$ ). ${ }^{\mathrm{g}}$ all 2-way interactions and $\mathrm{A}: \mathrm{B}: \mathrm{C}$ and $\mathrm{A}: \mathrm{C}: \mathrm{D}$ only (residual $\mathrm{df}=2$, deviance $=2.33$; ${ }^{\mathrm{h}}$ all 2-way interactions except $\mathrm{B}: \mathrm{C}$ (residual $\mathrm{df}=5$, deviance $=5.50$ ); ${ }^{\text {all }}$ 2-way interactions and A:B:C and B:C:D only (residual $\mathrm{df}=2$, deviance $=0.42$ ). $\mathrm{A}=\mathrm{HCV}$-Diagnosis, $\mathrm{B}=\mathrm{HIV}-\mathrm{Test}, \mathrm{C}=\mathrm{SMR} 01 /$ Deaths, and $\mathrm{D}=\mathrm{SDMD}$. 